\title{
Data Conveyance Maximization in Bilateral Relay System using Optimal Time Assignment
}

\author{
Dr. Joy Iong Zong Chen, \\ Professor, \\ Department of Electrical Engineering, \\ Da-Yeh University,Taiwan. \\ Email id: jchen@mail.dyu.edu.tw \\ Kong-Long Lai, \\ Department of Electrical Engineering, \\ Da-Yeh University, \\ No. 168 University Rd., \\ Dacun, Changhua 51591, \\ Taiwan
}

\begin{abstract}
The paper puts forward an optimal time assignment for the bilateral relay system in order to heighten the total amount of data conveyed through it. To have an optimal time assignment the conveyance rate of the two way transmission is obtained using the successful conveyance probability and the destined data rate. Taking into consideration the acquired particulars of data conveyance the paper has proposed an optimal time assignment for every connection. The results observed on evaluating the performance proved that the bilateral relay system with the optimal time assignments has improved the coverage, reliability and the throughput of the wireless communication system.
\end{abstract}

Keywords: Wireless Communication System, Bilateral Relay System, Data Conveyance Rate, Optimal Time Assignment, Performance Improvement

\section{Introduction}

The broadcasting system nowadays has become predominant in small cells technology that is put forth as a remedy to assist the demands of the fifth generation wireless system. In the broadcasting scheme, the relay is set up and activated in a wireless shadow arena where the data conveyance remains slightly complicated due to the interiors and the locations like undergrounds. But in traditional communication system every data point or the node engaged in conveying the information uses a dedicated relay completely

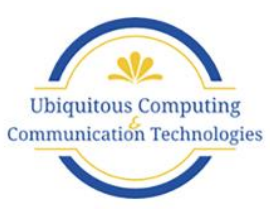


Journal of Ubiquitous Computing and Communication Technologies (UCCT) (2020)

Vol.02/ No. 02

Pages: $109-117$

https://www.irojournals.com/jucct/

DOI: https://doi.org/10.36548/jucct.2020.2.006

and additional duration is demanded for the relay to send and receive the information's. This minimizes the efficiency of the resources.

To eliminate the wastage of the resources the bilateral broadcasting system was introduced. In bilateral relaying, they were no dedicated relays and a single relay was shared across two data points to convey the information and enhance the resource efficiency eluding the wastage of resources. This remained profitable as it used only limited amount of relays in an efficient manner.

Despite the limited relay used in the bilateral relay system and the resource efficiency, its proper operation demands an efficient management of conveyance time (frame length). As the complete transmission time in the bilateral relays are limited, the complete time taken for conveyance is divided up for every conveyance of the user and the broadcast. In order to remove the unwanted conveyance time assignment that causes inadequacy in the conveyance time for other data points that requisites communication. The inadequacy in the conveyance time minimizes the total amount of data that are to be conveyed. Apart from this the traditional broadcasting methods are incompatible for the bilateral relay system as they operate using different protocols.

The inconsistency in the time assignment for the data transmission experienced in the prevailing researches are overcome in the proposed method. The time assignment put forward in the paper eludes the adaptive modulation followed in the previous method and designs a general optimal time assignment. By identifying the best values utilizing the numerical strategy. The proposed method improves the coverage, throughput and the reliability of the data conveyance and reduces the resource wastage by effectively managing the time of conveyance.

The remaining paper is organized with the literature survey on the prevailing methods and its demerits, in 2 , the proposed time assignment using the numerical scheme in 3 , the experimental evaluation analyzing the improvement in the throughput, coverage, reliability, of the data conveyance in the wireless system by the application of the proposed scheme in 4 and conclusion in 5.

\section{Literature Survey}

Taehoon, et al [1] has proposed a "duplex selection scheme for decoding and forwarding broadcast system to choose an improved duplex strategy based on the statistical conditions of the channel with a accurate

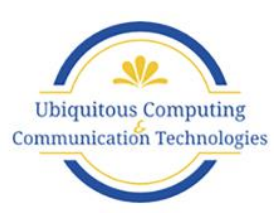


Journal of Ubiquitous Computing and Communication Technologies (UCCT) (2020)

Vol.02/ No. 02

Pages: $109-117$

https://www.irojournals.com/jucct/

DOI: https://doi.org/10.36548/jucct.2020.2.006

allocation of power. The results obtained though proves to have an reduced outage probability, it cause an enormous wastage of resources so proved to be inefficient"

Zhang et al [2] "the paper examines the potential full duplex techniques and incorporates a passive suppression, active analog and a digital withdrawal and presents the merits and the demerits, the paper also discuss the resulting end to end delay and the network congestion in the full duplex MAC protocol design. Further the outage probability of the multi-relay network was minimized substantially by introducing the Nakagami-m-fading channels in the context of the FD relaying"

Singh et al [3] has evaluated the "performance of the bilateral relay system using the analog network coding engaging the perspective of multitudes of parameters such as the overall symbol error rate, overall outage probability, ergodic sum rate, the paper examines the overall symbolic rate over the NM fading channel for a ANC and the variations of the overall symbolic rate with respect to the signal to noise ratio is observed."

Alouane et al [4] the author evaluates the incremental semi blind- opportunistic relay protocol in the bilateral broadcasting conveyance medium. The protocol is has significantly minimizes the cost and reduces the system performance of the system. Han et al [5] has proposed the "Performance bounds for two-way amplify-and-forward relaying."

Li et al [6] has performed the "Adaptive two-way relaying and outage analysis." Jitvanichphaibool, et al [7] has conducted the optimal resource allocation for two-way relay-assisted OFDMA." Zhang, et al [8] has proposed the "Resource allocation with subcarrier pairing in OFDMA two-way relay networks." Kumar, T. Senthil. et al [9] has put forth an "Efficient resource allocation and QOS enhancements of IoT with FOG network."

Valanarasu, Mr R et al [10] elaborates a "Comprehensive Survey of Wireless Cognitive and 5G Networks." Haoxiang, Wang et al [11] presents a "Multi-Objective Optimization Algorithm for Power Management in Cognitive Radio Networks." Smys et al [12] "Conducted emission reduction by frequency hopping spread spectrum techniques."Bhalaji, N. et al [13] performed a "Delay Diminished Efficient Task Scheduling and Allocation for Heterogeneous Cloud Environment."Manoharan, et al [14] devised the "Precision Improvement and Delay Reduction in Surgical Telerobotics."

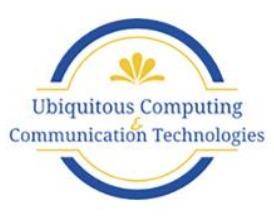


Journal of Ubiquitous Computing and Communication Technologies (UCCT) (2020)

Vol.02/ No. 02

Pages: $109-117$

https://www.irojournals.com/jucct/

DOI: https://doi.org/10.36548/jucct.2020.2.006

\section{Proposed Effective Conveyance Time Management}

A three data point circumstance is assumed for the proffered model that is based on the bilateral relaying system. Every consumer with the urgency to communicate is depicted as the user 1 and the user 2 and the data point for broadcasting is denoted as the relay data point 3. The $S N R_{x y}$ is the signal to noise ration measured from data point $\mathrm{x}$ to data point $\mathrm{y}$, and the fading channel are presumed to be "Rayleigh fading channel". During the first and the second stage the user 1 and user 2 start sending information's to the data point 3 and in the third step the data point 3 combines the conveys back the data to the each user. This make clear that the bilateral broadcasting minimizes the time and as well as the resources, unlike traditional broadcasting method that demands four steps. Where the user1data point sends the information to the relay, and the relay conveys it to the user 2 data point, now the user 2 data point does the same and the relay once again sends the information to the user 1 data point. The time assigned for the step I and II is denotes as $a^{t / 2}$ and the time assigned for the step III is denoted as $1-a^{t}$ where a is the assignment factor for time. The figure. 1 below depicts the system architecture bilateral relay system.

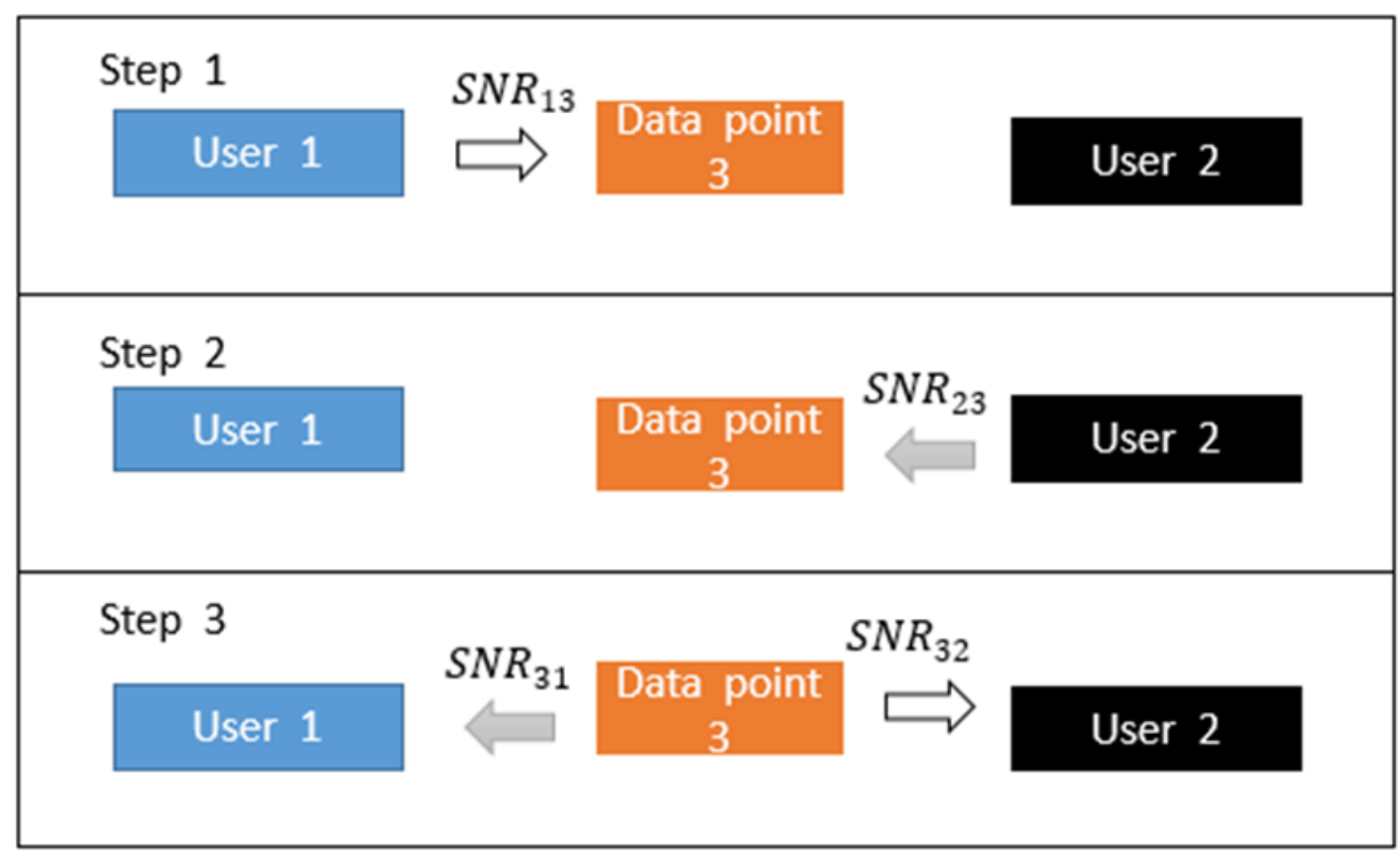

Figure.1 System Architecture for Bilateral Relay System 
Journal of Ubiquitous Computing and Communication Technologies (UCCT) (2020)

Vol.02/ No. 02

Pages: $109-117$

https://www.irojournals.com/jucct/

DOI: https://doi.org/10.36548/jucct.2020.2.006

\subsection{Average Data Rate of Conveyance}

To make the time assignment effective the data rate of the conveyance is derived. The conveyance rate is examined based on the "probability of successful transmission $\left(\operatorname{prob}\left(s_{d c}\right)\right)$ " and "target data rates $\left(T a_{d r}\right)$ " the $T a_{d r}$ between the user 1 and 2 to the data point 3 denoted as ' $T a_{d r 1-2}$ ', the $S N R_{12}$ to data point 3 is acquired to assist the $T a_{d r 1-2}$ as in (1) and (2)

$$
S N R_{13}=S N R_{23}=2^{T a_{d r 1-2}}-1
$$

Correspondingly,

$$
S N R_{31}=S N R_{32}=2^{2 T a_{d r 1-2}}-1
$$

The "outage probability" for every connection is defined as follows in the (3)

$$
\left\{\begin{array}{l}
O P_{1,3}=\operatorname{prob}\left(S N R_{13}<2^{T a_{d r 1-2}}-1\right. \\
O P_{2,3}=\operatorname{prob}\left(S N R_{23}<2^{T a_{d r 1-2}}-1\right. \\
O P_{3,1}=\operatorname{prob}\left(S N R_{31}<2^{2 T a_{d r 1-2}-1}\right. \\
O P_{3,2}=\operatorname{prob}\left(S N R_{32}<2^{2 T a_{d r 1-2}-1}\right.
\end{array}\right.
$$

As the fading channel are presumed to be "Rayleigh fading channel" the 'OP' for every link is given as in (4)

$$
\operatorname{Prob}_{x y}=\left\{\begin{array}{c}
1-e^{-\frac{T a_{d r 1-2}}{S N R_{x 3}}}, x=1,2 \\
1-e^{-\frac{2^{2 T a_{d r 1-2}-1}}{S N R_{3 y}}}, y=1,2
\end{array}\right.
$$

From this the number of successful transmissions rate $\left(s_{d c}\right)$ for every link is acquired using the (5)

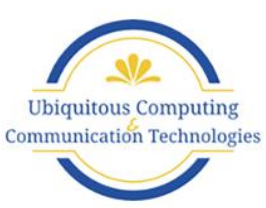


Journal of Ubiquitous Computing and Communication Technologies (UCCT) (2020)

Vol.02/ No. 02

Pages: $109-117$

https://www.irojournals.com/jucct/

DOI: https://doi.org/10.36548/jucct.2020.2.006

$$
s_{d c}(x, y)=\left\{\begin{array}{l}
T a_{d r 1-2}\left(1-O P_{x, 3}\right), x=1.2 \\
T a_{d r 1-2}\left(1-O P_{3, y}\right), y=1.2
\end{array}\right.
$$

As the count of data to be conveyed is proportional to the time assignment, due to the forwarding and the relaying procedure. It is estimated by the connection that is weak. The "average data transmission" is as follows.

Total $s_{d c}(a)=\min \left(\frac{a^{t} s_{d c}(1,3)}{2},(1-a) s_{d c}(3,2)\right)+\min \left(\frac{a^{t} s_{d c}(2,3)}{2},(1-a) s_{d c}(3,1)\right), 0 \leq a \leq 1$

Where min $(1,2)$ is the minimum value across 1 and 2.

The optimal time for assignment is estimated using the optimal assigning of time to maximize the total conveyance rate.

$$
\max _{a} \text { Total } s_{d c}(a), \text { subject to } 0 \leq a \leq 1
$$

\subsection{Assigning Optimal Time}

The optimal time are assigned using the following equation (8) and (9) shown the equation (6) can be maximized using the optimal time assignment.

$$
\begin{gathered}
a_{1}=\frac{2\left(1-O P_{3,2}\right)}{3-O P_{1,3}-2 O P_{3,2}}=\frac{2}{e^{\frac{2^{2 T a_{d r 1-2}-1}}{S N R_{32}}-\frac{T a_{d r 1-2}}{S N R_{1,3}}+2}} \\
a_{2}=\frac{2\left(1-O P_{3,1}\right)}{3-O P_{2,3}-2 O P_{3,1}}=\frac{2}{e^{\frac{2^{2 T a_{d r 1-2}-1}}{S N R_{31}}-\frac{T a_{d r 1-2}}{S N R_{2,3}}+2}}
\end{gathered}
$$


Journal of Ubiquitous Computing and Communication Technologies (UCCT) (2020)

Vol.02/ No. 02

Pages: $109-117$

https://www.irojournals.com/jucct/

DOI: https://doi.org/10.36548/jucct.2020.2.006

\section{Simulation Results}

The greater performance of the time assignment put forth in fig. 2 is verified comparing the complete data rate of conveyance for the optimal time assigned with normalized SNR is compared with the complete data rate of conveyance for the optimal time assigned using the numerical approach. The SNR normalized is not applied by the path-loss term. The exponent for the path loss is assumed as 4.5 , and the $T a_{d r 1-2}$ is assumed to be 1 . The relay localized at $.75, .5, .25$, between the node 1 and node 2 . The results observed depicts the assigned optimal time has maximized the Total $s_{d c}$

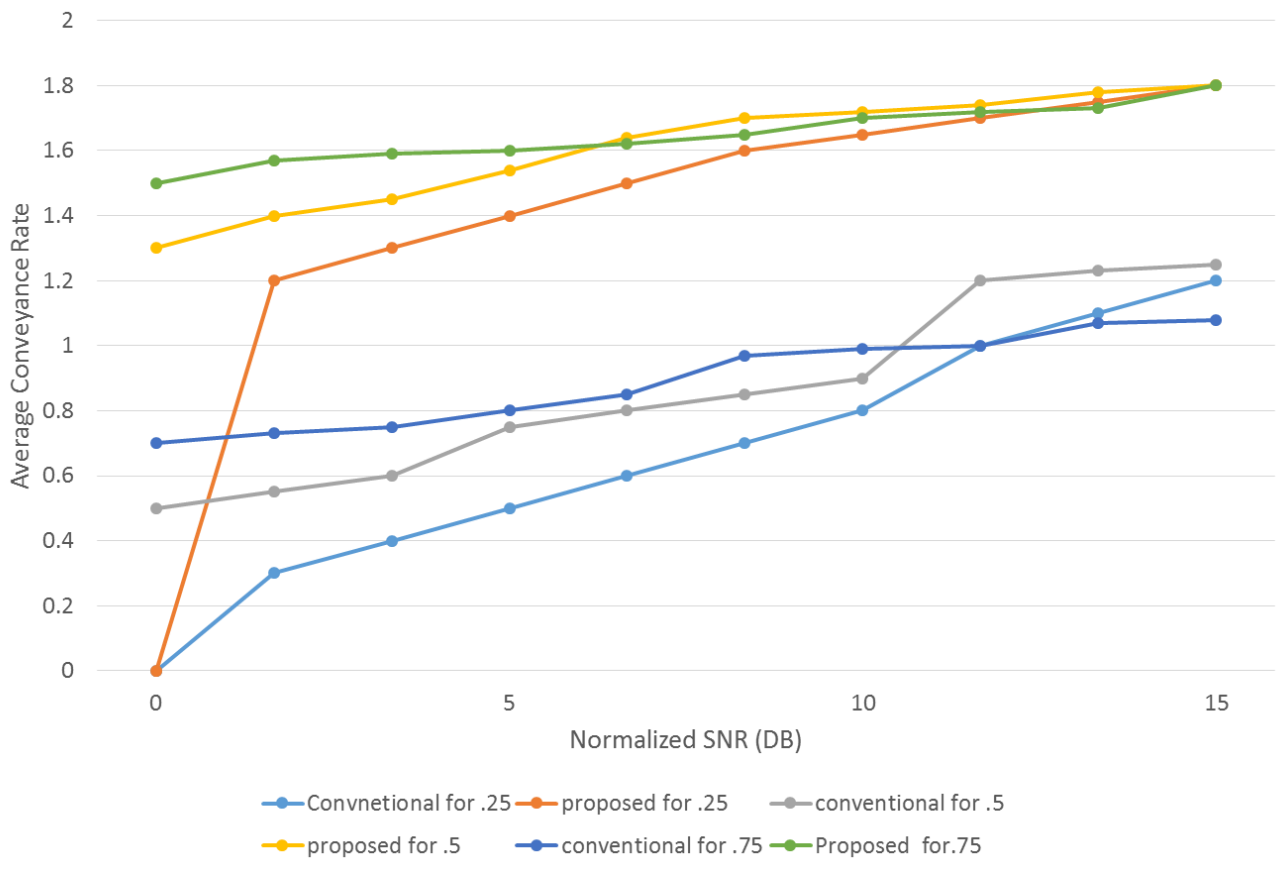

Figure. 2 Total $s_{d c}$ with the Normalized SNR

\section{Conclusion}

The assigning optimal time is appropriate way to maximize the rate of conveyance, the conveyance rate is extracted using the $\operatorname{prob}\left(s_{d c}\right)$ and the $T a_{d r}$ while using the bilateral relay system, according to the

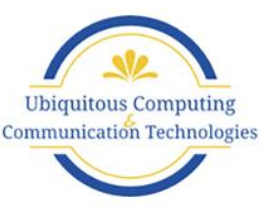


Journal of Ubiquitous Computing and Communication Technologies (UCCT) (2020)

Vol.02/ No. 02

Pages: $109-117$

https://www.irojournals.com/jucct/

DOI: https://doi.org/10.36548/jucct.2020.2.006

extracted information the optimal time assignment for the bilateral system is done and simulated. The simulation results demonstrate that the optimal time assignment maximizes the conveyance rate.

\section{References}

[1] Kwon, Taehoon, and Sungmook Lim. "Optimal Duplex Selection for Decode and Forward Relay Systems with Power Allocation." TIIS 10, no. 12 (2016): 5347-5360.

[2] Zhang, Zhongshan, Xiaomeng Chai, Keping Long, Athanasios V. Vasilakos, and Lajos Hanzo. "Full duplex techniques for 5G networks: self-interference cancellation, protocol design, and relay selection." IEEE Communications Magazine 53, no. 5 (2015): 128-137.

[3] Singh, Akshay Pratap, Anjana Jain, and Shekhar Sharma. "An Empirical Study of OSER Evaluation with SNR for Two-Way Relaying Scheme." In Intelligent Systems Technologies and Applications, pp. 67-74. Springer, Cham, 2016.

[4] Alouane, Wided Hadj, and Noureddine Hamdi. "Incremental Fixed-Gain Opportunistic AF in Two-Way Relaying Networks." Wireless Personal Communications 95, no. 2 (2017): 13731396.

[5] Han, Yang, See Ho Ting, Chin Keong Ho, and Woon Hau Chin. "Performance bounds for twoway amplify-and-forward relaying." IEEE Transactions on Wireless Communications 8, no. 1 (2009): 432-439.

[6] Li, Qiang, See Ho Ting, Ashish Pandharipande, and Yang Han. "Adaptive two-way relaying and outage analysis." IEEE Transactions on Wireless Communications 8, no. 6 (2009): 32883299.

[7] Jitvanichphaibool, Kommate, Rui Zhang, and Ying-Chang Liang. "Optimal resource allocation for two-way relay-assisted OFDMA." IEEE Transactions on Vehicular Technology 58, no. 7 (2009): 3311-3321.

[8] Zhang, Hao, Yuan Liu, and Meixia Tao. "Resource allocation with subcarrier pairing in OFDMA two-way relay networks." IEEE Wireless Communications Letters 1, no. 2 (2012): 61-64.

[9] Kumar, T. Senthil. "Efficient resource allocation and QOS enhancements of IoT with FOG network." J ISMAC 1 (2019): 101-110.

[10] Valanarasu, Mr R., and A. Christy. "Comprehensive Survey of Wireless Cognitive and 5G Networks." Journal of Ubiquitous Computing and Communication Technologies (UCCT) (2019): 23-32.

[11] Haoxiang, Wang. "Multi-Objective Optimization Algorithm for Power Management in Cognitive Radio Networks." Journal of Ubiquitous Computing and Communication Technologies (UCCT) 1, no. 02 (2019): 97-109.

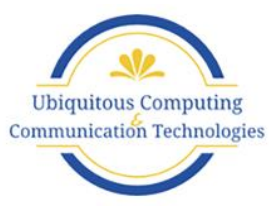


Journal of Ubiquitous Computing and Communication Technologies (UCCT) (2020)

Vol.02/ No. 02

Pages: $109-117$

https://www.irojournals.com/jucct/

DOI: https://doi.org/10.36548/jucct.2020.2.006

[12] Smys, Smys, J. Thara Prakash, and Jennifer S. Raj. "Conducted emission reduction by frequency hopping spread spectrum techniques." National Academy Science Letters 38, no. 3 (2015): 197-201.

[13] Bhalaji, N. "Delay Diminished Efficient Task Scheduling and Allocation for Heterogeneous Cloud Environment." Journal of trends in Computer Science and Smart technology (TCSST) 1, no. 01 (2019): 51-62.

[14] Manoharan, Samuel, and Narain Ponraj. "Precision Improvement and Delay Reduction in Surgical Telerobotics." Journal of Artificial Intelligence 1, no. 01 (2019): 28-36.

\section{Authors Biography}

Dr. Joy Iong-Zong Chen is currently a full professor of Department of Electrical Engineering Dayeh University at Changhua Taiwan. Prior to joining the Dayeh University, he worked at the Control Data Company (Taiwan) as a technical manager since Sep. 1985 toSep. 1996. His research interests include wireless communications, spread spectrum technical, OFDM systems, and wireless sensor networks. He has published a large number of SCI Journal papers in theissues addressed physical layer for wireless communication systems. Moreover, he also majors in developing some applications of the IOT (Internet of Thing) techniques and Dr. Joy I.-Z. Chen owned some patents authorized by the Taiwan Intellectual Property Office (TIPO)

Kong-Long Lai, is currently working as professor in the Department of Electrical Engineering, Da-Yeh University, No. 168 University Rd., Dacun, Changhua 51591, Taiwan. His area of research includes Information and communication technologies, Antenna design and RF propagation, Autonomic, \& Dependable computing, Green Computing, Computational Complexity and Networks

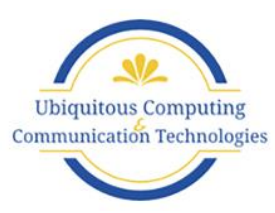

